\title{
Gravitationally lensed radio emission associated with SMM J16359+6612, a multiply imaged submillimeter galaxy behind A 2218
}

\author{
M. A. Garrett ${ }^{1}$, K. K. Knudsen ${ }^{2}$, and P. P. van der Werf ${ }^{3}$ \\ 1 Joint Institute for VLBI in Europe, Postbus 2, 7990 AA, Dwingeloo, The Netherlands \\ e-mail: garrett@jive.nl \\ 2 Max-Planck-Institute für Astronomie, Königstuhl 17, 69117 Heidelberg, Germany \\ 3 University of Leiden, Department of Astronomy, PO Box 9513, 2300 RA Leiden, The Netherlands
}

Received 7 December 2004 / Accepted 8 January 2005

\begin{abstract}
We report the detection of discrete, lensed radio emission from the multiply imaged, $z=2.516$ submillimetre selected galaxy, SMM J16359+6612. All three images are detected in deep WSRT $1.4 \mathrm{GHz}$ and VLA $8.2 \mathrm{GHz}$ observations, and the radio positions are coincident with previous sub-mm SCUBA observations of this system. This is the widest separation lens system to be detected in the radio so far, and the first time that multiply imaged lensed radio emission has been detected from a star forming galaxy - all previous multiply-lensed radio systems being associated with radio-loud AGN. Taking into account the total magnification of $\sim 45$, the WSRT $1.4 \mathrm{GHz}$ observations suggest a star formation rate of $\sim 500 M_{\odot} \mathrm{yr}^{-1}$. The source has a steep radio spectrum $\alpha \sim-0.7$ and an intrinsic flux density of just 3 microJy at $8.2 \mathrm{GHz}$. Three other SCUBA sources in the field are also detected by the WSRT, including SMM J16359+66118, a singly imaged (and magnified) arclet at $z=1.034$. Higher resolution radio observations of SMM J16359+6612 (and other highly magnified star forming galaxies) provide a unique opportunity to study the general properties and radio morphology of intrinsically faint, distant and obscured star forming galaxies. They can also help to constrain the technical specification of next generation radio telescopes, such as the Square Kilometre Array.
\end{abstract}

Key words. gravitational lensing - galaxies: starburst - radio continuum: galaxies

\section{Introduction}

SMM J16359+6612 is a sub-millimetre galaxy (SMG) located at $z=2.516$ that is triply imaged and highly magnified by the core of a massive foreground cluster, Abell 2218 (Kneib et al. 2004; Knudsen 2004). The three lensed images of the background source are highly magnified (by factors of 22, 14 and 9) and the maximum image separation is $\sim 41$ arcsec. All three images are detected by the Submillimeter Common-User Bolometric Array (SCUBA) at 850 and 450 microns with the brightest image having a measured 850 micron flux density of $17 \mathrm{mJy}$ (Kneib et al. 2004). Recently, both Kneib et al. (2005) and Sheth et al. (2004) have observed molecular emission from the $\mathrm{CO}(3-2)$ line for each of the three images. There are two distinct velocity components in this line separated by $280 \mathrm{~km} \mathrm{~s}^{-1}$, suggesting a total dynamical mass of the galaxy of $1.5 \times 10^{10} M_{\odot}$. There is also a spatial offset of 1 arcsec between these two components and Kneib et al. (2005) argue, that the source is likely to be a merging system with the 2 nuclei separated by $3 \mathrm{kpc}$.

At radio wavelengths only the bright tail of the SMG population is usually detectable, so observations of highly magnified systems such as SMM J16359+6612, offer a unique opportunity to study the radio properties of one example of the faint sub-mJy, SMG population. Little is known about the nature of these faint SMG, despite the fact that they dominate (energetically) the cosmic far-infrared background (Knudsen 2004). The intrinsic (unlensed) sub-mm flux of SMM J16359+6612 is estimated to be $S_{850}=0.8 \mathrm{mJy}$ (placing it well below the confusion limit of normal blank field sub-mm surveys). If one assumes that the FIR-radio correlation holds for this high- $z$ source (e.g. Garrett 2002), the intrinsic flux density of any radio counter-part is estimated to be only a few microJy at $8.2 \mathrm{GHz}$. However, the magnification provided by the lens boosts the flux density of even the faintest image in SMM J16359+6612 by a factor of 9, suggesting that radio counterparts to the sub-mm sources associated with SMM J16359+6612 should be easily detectable in deep Very Large Array (VLA) and Westerbork Synthesis Radio Telescope (WSRT) images of the field. We note that singly imaged SMG, modestly amplified by foreground clusters, have already been detected at radio wavelengths e.g. Smail et al. (2000) and Ivison et al. (2001).

In this paper we present WSRT $1.4 \mathrm{GHz}$ and VLA $8.2 \mathrm{GHz}$ observations of Abell 2218, specifically the area of sky surveyed by the Leiden-SCUBA lens survey (Knudsen 2004) that 
includes SMM J16359+6612. The VLA data are in the public domain and were extracted from the on-line VLA archive. In Sect. 2 we describe the radio observations and data analysis. The results and discussion are presented in Sect. 3 and we give our conclusions in Sect. 4. Throughout this paper we assume an $\Omega=0.3, \Lambda=0.7$ cosmology with $H_{0}=70 \mathrm{~km} \mathrm{~s}^{-1} \mathrm{Mpc}^{-1}$.

\section{Radio observations}

\subsection{WSRT observations}

Observations of Abell 2218 at $1.4 \mathrm{GHz}$ were conducted by the WSRT on 5 November 2004. The 12 hour run used the full 160 $(8 \times 20) \mathrm{MHz}$ WSRT observing band and employed the default continuum frequency set-up (with the eight bands centered between 1.311 and $1.450 \mathrm{GHz}$ ). Observations were made assuming a coordinate equinox of 2000. For each of the 8 bands, 64 spectral channels were generated (a total of 512 spectral points were obtained for the $160 \mathrm{MHz}$ band) and 4 polarization products were recorded. Unfortunately, radio telescope "RT7" was being used for single-dish VLBI observations and was unavailable during the entirety of these observations. A short 20-min scan on 3C 48 was used to amplitude calibrate the data. A phase reference source $(\mathrm{J} 1642+689)$ was observed for 5 min every hour. The data analysis was performed using the NRAO AIPS package. The fringe-fitted phase solutions from J1642+689 were applied to the A 2218 target field. For this field, the entire WSRT primary beam was imaged using the AIPS task IMAGR. Several bright sources were detected in the field of view, and the data were self-calibrated using these and other sources in the field as a sky model. These outlying sources were later subtracted from the data set and self-calibration continued using only those sources located in the centre of the field. The data were self-calibrated first in phase and later in both amplitude and phase using the AIPS task CALIB.

A uniformly weighted ("robust -1 ") WSRT image of the field convolved with a Gaussian restoring beam of $13.6 \times$ 12.4 arcseconds (in position angle, $\mathrm{PA}=-0.21^{\circ}$ ) is shown in Fig. 1. At $1.4 \mathrm{GHz}$ there is a great deal of extended emission in the vicinity of the A 2218 cluster core. In order to enhance the contrast of the WSRT image, spacings shorter than $800 \lambda$ were not used to form the image presented in Fig. 1. The image reaches a $1 \sigma \mathrm{rms}$ noise level of $\sim 15 \mu \mathrm{Jy}_{\text {beam }^{-1}}$.

\subsection{VLA observations}

We searched the VLA archive for observations associated with the central area of Abell 2218. Several data sets were identified that included short, snapshot observations of this field. Only one full-track $8.2 \mathrm{GHz}$ observation of A 2218 was identified, AR045. These $X$-band observations were made on 1999 June 18 UT with the VLA in its D $(3 \mathrm{~km})$ configuration. Data were acquired in dual circular polarizations with bandwidths of $25 \mathrm{MHz}$ and at center frequencies of 8.1732, and 8.2732 GHz. Each of these $25 \mathrm{MHz}$ IFs was further divided into seven spectral channels with a width of $3.125 \mathrm{MHz}$. Observations were made assuming a coordinate equinox of 2000. The absolute

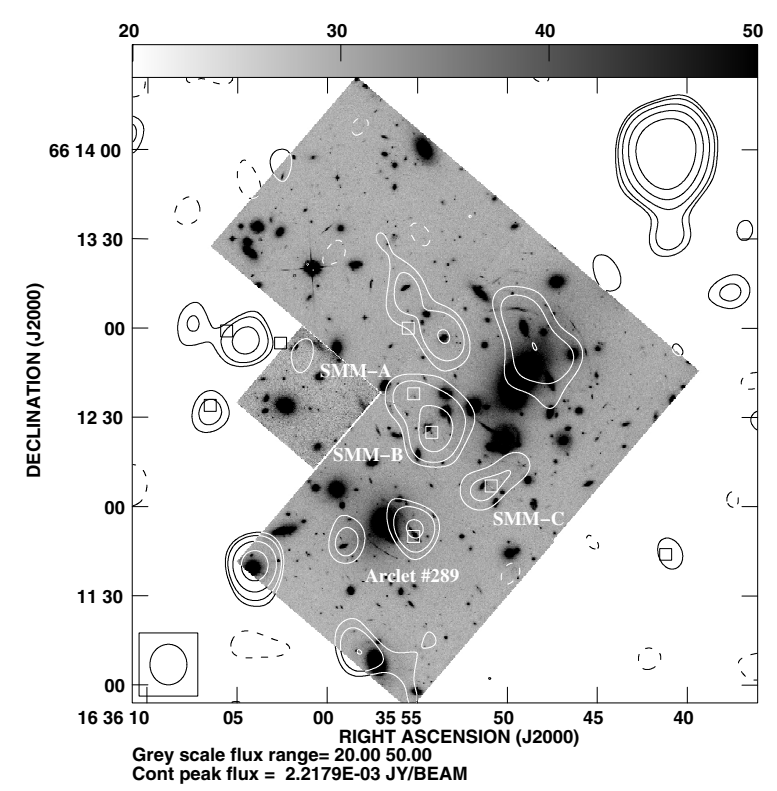

Fig. 1. The WSRT $1.4 \mathrm{GHz}$ contour map superimposed upon an HST F702W image of the region of sky associated with SMM J16359+6612. Radio emission from all three lensed images (SMM-A, SMM-B and SMM-C) is detected by the WSRT. The boxes represent the SCUBA source positions. Radio emission from three other SCUBA sources in the field (including the highly magnified arclet \#289, SMM J16359+66118) are also boxed. Contours are drawn at $-3,3,5,10,20$ and 40 times the $1-\sigma$ noise level of 15 microJy/beam.

flux density scale was set by observations of 3C 286 . The data calibration was made in the standard way with the phase variations during the observations calibrated via short observations of the VLA calibrator source $1642+689$. Unfortunately observations of $1642+689$ were only made 3 times over the course of the $24 \mathrm{~h}$ observations. The calibrated A 2218 data were clipped in order to remove a few high points before beginning the selfcalibration process. The self-calibration process followed the same outline as that described for the WSRT observations.

A naturally weighted VLA image of the field convolved with a Gaussian restoring beam of $11.2 \times 10.4$ arcsec (in position angle, $\mathrm{PA}=-72^{\circ}$ ) is shown in Fig. 2. The image has an rms noise level of 6 microJy beam.

\section{Results and discussion}

Figures 1 and 2 present the uniformly weighted WSRT and naturally weighted VLA contour maps of part of the A 2218 field, superimposed upon an HST F702W image (Kneib et al. 1996) of the region of sky that includes the lensed sub-mm galaxy SMM J16359+6612 (Kneib et al. 2004). The positions of the nine sources detected by SCUBA at $850 \mathrm{mi}-$ cron (Knudsen 2004) are also identified as square boxes in these figures. Following Kneib et al. (2004) we identify the lensed images as SMM-A (SMM J16359+6612.6), SMM-B (SMM J16359+6612.4) and SMM-C (SMM J16358+6612.1). Both the WSRT and VLA observations detect radio emission associated with all three lensed images. The faintest SCUBA detection (SMM-C) is detected at the 4-sigma level by the VLA at $8.2 \mathrm{GHz}$ and at the 7-sigma level by the WSRT at $1.4 \mathrm{GHz}$. 
Table 1. Details of the WSRT and VLA radio sources associated with SMM J16359+6612 (SMM-A, B, C) and SMM J16359+66118 (arclet \#289).

\begin{tabular}{|c|c|c|c|c|c|c|c|}
\hline Name & $\begin{array}{c}\mathrm{RA}\left(+16^{\mathrm{h}} 35^{\mathrm{m}}\right) \\
\mathrm{J} 2000(\mathrm{~s})\end{array}$ & $\begin{array}{l}\operatorname{Dec}\left(+66^{\circ}\right) \\
J 2000\left({ }^{\prime},{ }^{\prime \prime}\right)\end{array}$ & $\begin{array}{l}S_{\mathrm{T}} \\
\mu \mathrm{Jy}\end{array}$ & $\begin{array}{l}S_{\mathrm{Pk}} \\
\mu \mathrm{Jy}\end{array}$ & $\begin{array}{c}\text { Maj axis } \\
\text { " }\end{array}$ & $\begin{array}{c}\text { Min axis } \\
\prime \prime\end{array}$ & $\begin{array}{l}\text { PA } \\
\text { deg }\end{array}$ \\
\hline WSRT J163550+661205 (SMM-C) & $51.1 \pm 0.1$ & $12,08.0 \pm 1.0$ & $110 \pm 17$ & $110 \pm 17$ & - & - & - \\
\hline WSRT J163553+661226 (SMM-B) & $53.9 \pm 0.1$ & $12,26.4 \pm 0.6$ & $391 \pm 43$ & $217 \pm 16$ & $15 \pm 2$ & $9 \pm 2$ & $165 \pm 12$ \\
\hline WSRT J163555+661237 (SMM-A) & $55.6 \pm 0.2$ & $12,37.7 \pm 1.0$ & $99 \pm 17$ & $99 \pm 17$ & - & - & - \\
\hline VLA J163550+661206 (SMM-C) & $50.5 \pm 0.2$ & $12,06.8 \pm 0.1$ & $24 \pm 10$ & $24 \pm 6$ & - & - & - \\
\hline VLA J163554+661223 (SMM-B) & $54.5 \pm 0.1$ & $12,25.3 \pm 1.1$ & $124 \pm 18$ & $51 \pm 6$ & $16 \pm 3$ & $10 \pm 3$ & $39 \pm 12$ \\
\hline VLA J163555+661236 (SMM-A) & $55.7 \pm 0.1$ & $12,37.1 \pm 0.5$ & $28 \pm 6$ & $28 \pm 6$ & - & - & - \\
\hline WSRT J163555+661152 (\#289) & $55.2 \pm 0.1$ & $11,52.9 \pm 0.6$ & $170 \pm 29$ & $175 \pm 17$ & $6 \pm 4$ & - & $43 \pm 20$ \\
\hline VLA J163555+661150 (\#289) & $55.1 \pm 0.2$ & $11,50.1 \pm 0.6$ & $35 \pm 10$ & $37 \pm 6$ & $8 \pm 3$ & - & $65 \pm 10$ \\
\hline
\end{tabular}

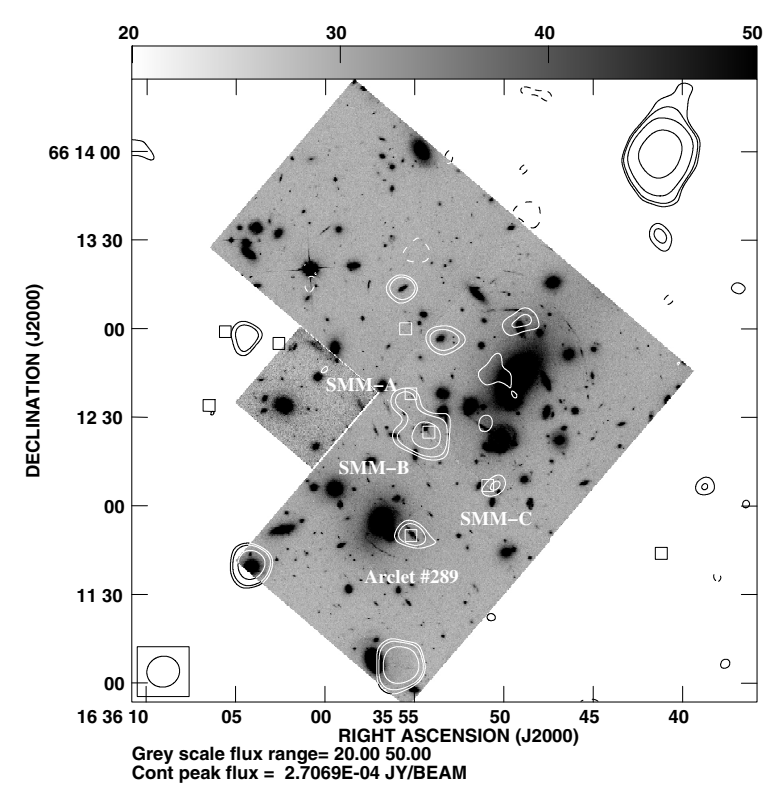

Fig. 2. The VLA $8.2 \mathrm{GHz}$ contour map superimposed upon an HST F702W image of the region of sky associated with SMM J16359+6612F702W. Radio emission from all three lensed images is detected by the WSRT. The boxes represent the SCUBA source positions. Radio emission from the highly magnified arclet (\#289), SMM J16359+66118 is also detected. Contours are drawn at $-3,3,4$, 7 and 10 times the $1-\sigma$ noise level of 6 microJy/beam.

The AIPS task IMFIT was used to fit Gaussian components to the WSRT and VLA radio sources. Details of the radio source's total flux density $\left(S_{\mathrm{T}}\right)$, peak flux density $\left(S_{\mathrm{Pk}}\right)$, position and (where appropriate) deconvolved Gaussian sizes (major axis, minor axis and position angle) are detailed in Table 1, together with their associated formal errors. It was not possible to robustly constrain the deconvolved size of the Gaussian component associated with the fainter images SMM-A and SMM-C in the VLA and WSRT images. For SMM-A and SMM-C the Guassian component size was thus fixed to the respective restoring beam sizes. For both the VLA and WSRT images, two Gaussians were fitted to the SMM-A,B region simultaneously.

The SCUBA, VLA and WSRT observations have comparable resolution. The error in the SCUBA 850 micron positions (including confusion effects) is expected to be $\sim 4$ arcsec (Knudsen 2004). The radio positions are expected to be better than this, with an accuracy of 2 arcsec or better. Slightly larger errors are possible for the radio positions derived by the WSRT, due to mild confusion in the field. The radio and sub-mm positions of SMM-A, B, C are all consistent within the errors - the largest deviation is an offset of 3 arcsec (the VLA/SCUBA comparison in the case of the faintest image SMM-A). There are no obvious systematic offsets in the sub-mm and radio positions.

At $8.2 \mathrm{GHz}$ the total flux density of SMM-B is 124 microJy, somewhat larger than our original expectations (see Sect. 1). By co-adding the total flux density of all three images at 1.4 and $8.2 \mathrm{GHz}$, we derive a spectral index of the source $\alpha=-0.7$, similar to the steep values measured by Richards (2000) for star forming galaxies in the Hubble Deep Field North. Kneib et al. (2005) have shown that the overall SED of SMM J16359+6612 is similar to Arp 220. By adopting an Arp 220 SED, we estimate the source's k-corrected radio luminosity at $1.4 \mathrm{GHz}$ to be $\sim 4 \times 10^{23}$ Watts/Hz. Using the relation between Star formation rate (SFR) and radio luminosity (Condon 1992), we derive an intrinsic star formation rate (SFR) for SMM J16359+6612 of $500 M_{\odot} \mathrm{yr}^{-1}$. This is in good agreement with the value obtained by Kneib et al. (2004) from the FIR luminosity. As noted by Kneib et al. the uncorrected $\mathrm{H} \alpha$ SFR estimate is only $11 M_{\odot} \mathrm{yr}^{-1}$, suggesting that this galaxy is highly obscured by dust.

The VLA 8.2 GHz observations best resolve the lensed images. The flux density ratio of the images at $8.2 \mathrm{GHz}$ (and their formal error) are $\sim 0.23 \pm 0.06$ and $\sim 0.19 \pm 0.08$ for SMM-A/SMM-B and SMM-C/SMM-B respectively. Similarly, the WSRT $1.4 \mathrm{GHz}$ flux image density ratios are $0.25 \pm 0.05$ and $0.28 \pm 0.06$ respectively. The radio flux density measurements at 1.4 and $8.2 \mathrm{GHz}$ are thus consistent with the source being gravitationally lensed. In comparison, the SCUBA observations give higher values of 0.64 and 0.53 , the IRAM CO(3-2) line intensities (Kneib et al. 2005) give 0.67 and 0.63 , and the $K$-band observations (Kneib et al. 2004) give 0.53 and 0.4. Lens models of this system (based on Kneib et al. 1996), predict flux density ratios of 0.63 and 0.4 . Discrepancies in the flux density ratio at different wavelengths might be explained 
if the various emission regions are not co-located or have very different size-scales over which the magnification may change. We also note that the flux density ratio of SMM-A, B and C between 850 micron and $1.4 \mathrm{GHz}$ are $111 \pm 22,43 \pm 7$ and $82 \pm 16$. By comparison the 850 micron $/ 1.4 \mathrm{GHz}$ flux density ratio of another SMG also located at $z \sim 2.5$, SMM J14011+0252 (Ivison et al. 2000) is $127 \pm 37$. It may be that in the case of SMM J16359+6612, the discordant flux ratios at both 1.4 and $8.2 \mathrm{GHz}$ can be explained by enhanced radio emission associated with SMM-B, perhaps from another radio source also located in this region of the field. Higher resolution radio observations are required in order to settle this question.

As shown in Table 1, the brightest image SMM-B is resolved by the VLA $8.2 \mathrm{GHz}$ observations. The measured PA of the major axis is $\sim 39$ degrees, in good agreement with the position angle of the major axis of the $\mathrm{CO}(3-2)$ measurements of the same component, $\sim \mathrm{PA}=30 \mathrm{deg}$. Both measurements are consistent with the overall extension of the associated arc-like HST images (Kneib et al. 2004). The FWHM of the major axis of the SMM-B (see Table 1) does not exceed $\sim 17$ arcsec. With a magnification of $\sim 22$ for this image, the intrinsic size must be on the arcsecond or sub-arcsecond scale - consistent with the sub-galactic sizes measured for radio sources in the HDF-N.

The WSRT observations also detect other radio counterparts to other sub-mm sources detected by SCUBA in this field (Knudsen 2004). In particular, we detect radio counter-parts to SMM J16357+66117 (4o detection), SMM J16361+66126 and SMM J16359+66118. The latter source (see Table 1) is previously identified by Kneib et al. (1996) as a $z=$ 1.034 singly-imaged lensed arc (also known as arc \#289, see Swinbank et al. 2003, and references therein) with a magnification of $\sim 7$. The source is also detected by the $8.2 \mathrm{GHz}$ VLA observations presented here, and by the ISOCAM at 15 micron (Barvainis et al. 1999). In both the WSRT and VLA images, the measured size of the arclet is less than the restoring beam, in particular the minor axes of the fitted Gaussians are unresolved. The position angle of the major axes are consistent with the optical extension of this system. This source also has a steep spectral index, $\alpha \sim 0.9$. The VLA 8.2 GHz observations do not detect the other SCUBA sources - SMM J16357+66117 and SMM J16361+66126. These are fainter than SMM J16359+66118, and must have fairly steep spectral indices $(\alpha>0.5)$. Further details of the radio counterparts to these SCUBA sources will be reported elsewhere.

\section{Conclusions}

We have detected with the WSRT at $1.4 \mathrm{GHz}$ and VLA at $8.2 \mathrm{GHz}$, radio emission associated with the triply lensed SMG, SMM J16359+6612. This is the first time that radio emission has been detected in a multiply imaged SMG system lensed by a foreground cluster. The maximum image separation is $\sim 41$ arcsec, much larger than any other lens system detected in the radio. This is also the first time that multiply lensed radio emission has been detected from a star forming galaxy - all previous multiply imaged radio lensed systems are associated with radio-loud AGN. The properties of the three radio sources are largely consistent with the gravitational lensing hypothesis. In addition to detecting SMM J16359+6612, we also detect radio emission coincident with SMM J16359+66118 a singly imaged arclet (\#289). The sources are only detectable by current radio instruments due to the high magnification factor provided by the lens ( $\sim 45$ in the case of SMM J16359+6612). The intrinsic total flux density of the radio source at 1.4 and $8.2 \mathrm{GHz}$ is $\sim 14$ and $\sim 3$ microJy.

Follow-up radio observations of SMM J16359+6612 with much better angular resolution than those presented here are warranted. Since sub-arcsecond imaging is routinely possible at radio wavelengths, it should be possible to fully capitalise on A 2218 as a "natural telescope". A comparison with the detailed HST images of this system will be particularly interesting. Measurements of the size and a study of the morphology of the radio emitting region should also constrain the extent of the star formation in this galaxy. Upgraded radio telescopes (such as the EVLA and e-MERLIN) should be able to detect many more highly magnified lens systems lying behind massive foreground clusters. In very deep integrations, the eMERLIN and the EVLA telescopes now under development, may be able to detect radio sources associated with individual SNe (or SNR), assuming these have luminosities similar to those detected in local mergers, such as Arp 220. Radio observations of SMM J16359+6612 and other highly magnified SMG using existing and upgraded radio telescope facilities, will be important in determining the general radio properties (e.g. angular size) of the faint SMG population, providing essential input to the technical specification of next generation instruments, such as the Square Kilometre Array.

Acknowledgements. We would like to thank the staff of the WSRT including Rene Vermeulen, Raffaella Morganti \& Willem Baan who helped make these observations possible at very short notice. We also thank the referee, J.-P. Kneib for useful comments and suggestions, that have improved the paper. The WSRT is operated by ASTRON (The Netherlands Foundation for Research in Astronomy) with support from The Netherlands Foundation for Scientific Research (NWO). The National Radio Astronomy Observatory is a facility of the National Science Foundation operated under cooperative agreement by Associated Universities, Inc. This work was supported in part by the European Communitys's Sixth Framework Marie Curie Research Training Network Programme, Contract No. MRTN-CT2004-505183 "ANGLES".

\section{References}

Barvainis, R., Antonucci, R., \& Helou, G. 1999, AJ, 118, 645

Condon, J. J. 1992, ARA\&A, 30, 575

Garrett, M. A. 2002, A\&A, 384, L19-22

Ivison, R., Smail, I., Frayer, D. T., et al. 2001, ApJ, 561, L45

Kneib, J.-P., Ellis, R., Smail, et al. 1996, ApJ, 471, 643

Kneib, J.-P., van der Werf, P. P., Knudsen, K. K., et al. 2004, MNRAS, 349,1211

Kneib, J.-P., Neri, R., Smail, I., et al. 2005, A\&A, accepted [arXiv: astro-ph/0409502]

Knudsen 2004, Ph.D. Thesis (Leiden University)

Richards 2000, ApJ, 533, 611

Sheth, K., Blain, A. W., Kneib, J.-P., et al. 2004, ApJ, 614, L5-8

Smail, I., Ivison, R. J., Owen, F. N., et al. 2000, ApJ, 528, 612

Swinbank, A. M., Smith, J., Bower, R. G., et al. 2003, ApJ, 598, 162 